\title{
Performance of A Global Circuit-Switched Satellite Communication Network*
}

\author{
Zsehong Tsai, Chuan-Chen Chuang, Jin-Fu Chang ${ }^{\dagger}$ Chun-ming Huang \\ Dept. of Electrical Engineering \\ National Taiwan University \\ Taipei, Taiwan, R.O.C.
}

\begin{abstract}
A circuit-switched network consisting of multiple low earth orbiting satellites and three geostationary satellites is considered. Crosslinks among low-orbit satellites are used as the major communication channels, while calls are routed via geostationary satellites only when low-orbit routes exceed or equal to a hop-count threshold. Simulation results are used to illustrate the network throughput under various traffic conditions and different link capacities. The impact of satellite failures on the network performance is also investigated.

Keywords: Network, Circuit-Switched
\end{abstract}

\section{INTRODUCTION}

Although almost all operational satellite communication networks employ geostationary(GEO) satellites, there has been increased interest in the networking techniques using multiple low earth orbiting(LEO) satellites in recent years[1]-[4]. Since the orbits of these LEO satellites are much lower in altitude than the GEO satellites, the footprints of LEO satellites are also smaller. Multiple LEO satellites, which can be in different orbits, are thus required to provide sufficient coverage for global communications. For example, in the Iridium system[2] 77 satellites are used and the global message network proposed in [4] requires 30 satellites. Usually, several orbits are employed in a low-orbit satellite network and each orbit is shared by a number of uniformly distributed satellites. In such a constellation, crosslinks can be established between two neighboring low-orbit satellites, which can be on the same or different orbits. Although low-orbit satellites are in continuous motion and their relative locations could change in time, inter-satellite crosslinks can still be

*This work was supported by National Science Council under grant NSC80-E-SP-002-D2.

tCurrently with Department of Electrical Engineering, National Central University, Chung-li, Taiwan, R.O.C. maintained for periods long enough for steady and stable communications[2], [4].

Due to the high connectivity of low-orbit satellite networks, these networks are expected to provide high survivability and flexibility. That is, a single intermediate-node failure or a link congestion event should not lead to total disruption of communications between any source-destination pair of satellites, because a large number of routes should be available within the network. Therefore, the operations of such networks should be flexible enough to support various groups of users for reliable communications. In order to meet the high expectations in network reliability, proper routing policies are thus essential to the operation of low-orbit satellite networks.

Another important feature of LEO satellite networks is the low power requirement for the communications between low-orbit satellites and ground stations, for both uplinks and downlinks. This new feature is due to the fact that the altitudes of LEO satellites are much lower than those of GEO satellites. Mobile stations are thus easier to be supported by such low-orbit satellite networks for global communications, although the addressing of mobile users have become a new problem. A recent proposal of the low-orbit cellular communication network can be found in [2].

In this paper, we propose a new satellite network architecture employing multiple low-orbit satellites and three geostationary satellites. We assume circuit-switching operations and sufficient on-board processing capability for call routing on all satellites, since voice communications is expected to play a major role in the low-orbit cellular communications environment. This architecture is designed to further improve the survivability and flexibility of low-orbit satellite network designs, due to the following observations. As noted in previous studies on circuit-switched networks, alternate routing policies could assign traffic streams to routes with large hop counts and lead to congestions under heavy traffic conditions[5]. Therefore, if these long-distance call$s$ are routed via GEO satellites, the traffic load on LEO satellites could be decreased significantly and 
these calls are able to avoid those congested links on the low-orbit satellite network. Blocking probability for calls requiring small hop count routes can also be reduced. Since the on-board processing capability on satellites can be much more restrictive than circuit switches in regular telecommunication networks[6], the use of GEO satellites can help to lower the processing load on LEO satellites and shorten the call set up time. In this paper, an alternate routing algorithm is especially designed to distribute long distance traffic to GEO satellites under the proposed multiple LEO-GEO satellite network architecture.

In section 2, we describe the network system configuration and the call setup procedure, including the addressee finding procedure and alternate routing policy. In section 3 , we present the network performance results obtained via simulations. Based on an Iridium-like configuration, different link capacities, traffic loads, and satellite failure conditions are considered. Conclusions are then provided in section 4.

\section{SYSTEM CONFIGURATION AND ROUTING POLICY}

We consider an Iridium-like configuration with 77 LEO satellites and 3 GEO satellites. The LEO satellites follow the orbits described in [2], while the GEO satellites are assumed to be 120 degrees apart. Each LEO satellite uses 6 crosslinks to communicate with its 6 neighboring LEO satellites, 2 on the same orbit and 4 on neighboring orbits. In addition, each LEO satellite is allowed to setup a crosslink with exactly one GEO satellite, while three GEO satellites use 3 separate crosslinks for their own communications. For convenience, we shall also call this network the LEO-GEO satellite network in the rest of this paper.

In the LEO-GEO network, each satellite can serve as a circuit switch and all cross links have part of the bandwidth reserved for circuit-switched calls. We assume that unreserved bandwidth of the crosslinks can be used for packet communications, including the signaling packets of circuit-switching operations. Since there can be many uplinks and downlinks between earth stations and an LEO satellite, these links are assumed to be of sufficient capacity for up/down link communications. We also assume the channel capacity for packet communications is always sufficient such that the signaling/data packet traffic are well supported.

Two types of users are assumed in our network architecture: users on local telecommunication networks and users on mobile stations. We use a various number of ground gateways as the in terworking units between the satellite network and ground $t$ elecommunication networks. These gateways also serve as databases for the location information of mobile users who directly communicate with LEO satellites. During the call setup procedure, the location of non-mobile user is fixed and only the routing operation is required. In turn, the location of a mobile station can be time dependent. An addressee finding procedure is required when a call is destined to a mobile user.

In [1], Brayer has proposed an addressee finding procedure in which a broadcasting-then-paging technique is used. This technique should be easy to implement but might generate heavy traffic on the signaling channels. With the deployment of ground gateways, we propose the use of the following procedure. In our system, each mobile station is required to register at its home gateway and an entry regarding its current location is created in the database. When a mobile station moves to a different area, the mobile station should notify the local gateway by sending registration messages periodically, via LEO satellites. The local gateway is then responsible to send the new address of this mobile user back to its home gateway. When such registrations and database updates are completed, the addressee finding at the call setup time should require only simple inquiry operations at the home gateway database.

The routing policy employed in our LEO-GEO satellite network is a variation of alternate routing used in traditional circuit-switched telecommunication networks[6]. Crosslinks among low-orbit satellites are used as the major communication channels, while calls are routed via geostationary satellites only when low-orbit routes exceed a hop-count threshold. During the call setup procedure, the shortest path between the source-destination LEO satellite pair is searched, considering only the crosslinks of LEO satellites. The shortest path of LEO crosslinks is selected if all of its links are still of residual capacity; otherwise the second shortest path is searched. During the search procedure, if two or more candidate paths are found to be the shortest (or the second shortest), the one with larger residual capacity is selected. The hop counts of the shortest as well as the second shortest path are checked for distance threshold violations. When the violation occurs, the path via GEO satellite(s) is then selected. Such a GEO path can make use of one or two GEO satellites, depending on the locations of the source-destination LEO satellite pair. If the selected path for a call is found to be still out of capacity, i.e. any of its links is full, then this call is blocked. A detail flow diagram for this routing policy is depicted in Fig.1.

Using the above described alternate routing policy, calls can be easily rerouted around any disrupted link or LEO satellite, by considering the latter to be under out-of-capacity conditions. That is, when a 
single link failure occurs, it can be considered as a special congestion condition by the routing algorithm. Only users currently located in the footprint of an out-of-service LEO satellite can be more seriously affected. As noted earlier, all LEO satellites are actually in fast motion. A single LEO satellite failure should only lead to communication disruption for a small period of time. In other words, we have the conjecture that high throughput of the network could still be maintained even under a small scale failure with our alternate routing policy and the multiple LEO-GEO satellite architecture. In the following, we shall illustrate the performance of the network under various traffic loads, with or without satellite failures.

\section{SIMULATION RESULTS}

During computer simulations of an experimental satellite network, the capacities of the following three types of intersatellite links are of importance: crosslinks of LEO satellites, crosslinks of GEO satellites, and links between GEO and LEO satellites. By assuming all links of the same type to be of the same capacity, we dramatically reduced the number of system parameters for our simulations. The up/down links between earth stations and the LEO satellites are assumed to be of infinite capacity since these links are less likely to be the bottlenecks of the network. Unless stated otherwise, all satellites and links are also assumed to be under normal operating conditions.

In the simulation, we also did not include the hand-off activities between LEO satellites, by assuming steady state and symmetric traffic conditions. Such an assumption is equivalent to having a static configuration and simplifies the network operations during simulations. We also assume the following traffic conditions for all LEO satellites: Poisson call arrival process, identical call arrival rate from ground stations, and uniform destination distributions. For simplicity, call durations are also assumed to be exponentially distributed.

The system parameters required for the network simulation are summerized as follows.

$\lambda$ - mean call arrival rate from earth stations for an LEO satellite.

$1 / \mu$ - average call holding time. This parameter is always set equal to 100 secs in the simulations.

$L E O \_C A P$ - capacity of crosslinks among lowearth orbiting satellites.

GEO_CAP - capacity of crosslinks among geostationary satellites.

$L G \_C A P$ - capacity of intersatellite links between LEO and GEO satellites.

threshold - the minimum hop count of an LEO path which needs to be rerouted via GEO satellites.
We first compare the call blocking probability of the proposed LEO-GEO satellite network with that of a similar satellite network consisting of LEO satellites only. The LEO satellites of both networks are of the same constellation and all crosslinks between LEO satellites are of the capacity for 5 calls. In our LEO-GEO satellite network, GEO_CAP is set equal to $25, L G \_C A P$ is set to be 5 and threshold is 6 . The offered network traffic load, equal to $77 \lambda / \mu$, is varying. All parameters are selected only to simulate network congestion conditions, instead of a practical design. In Figure 2, our proposed LEO-GEO network is found to provide better performance under any offered network traffic load, as one might expect.

In order to achieve higher network throughput, (or equivalently lower call blocking probability), proper selection of the threshold in the routing algorithm is essential. In Figure 3, we illustrate the impact of the threshold value on the network performance. With $L E O \_C A P$ equal to 5 , total network traffic load equal to 1000 Erlangs, $G E O \_C A P$ ranging from 25 to 150 , and $L G \_C A P=1 / 5 G E O \_C A P$, the optimal threshold values are all found to be 4 under this particular traffic condition. We note that the optimal threshold parameter is actually dependent on the traffic condition. Since traffic fluctuation is common in most networks, tuning of this routing parameter is necessary for keeping the LEOGEO satellite network under a near-optimal operating condition.

By conducting detail simulations of various threshold values and traffic loads, we plot the optimal thresholds versus network traffic load in Figure 4(a) and 4(b), using two experimental network configurations. In both configurations, crosslinks among GEO satellites are assumed to be of the capacity for 75 calls, while crosslinks between GEO and LEO satellites are of the capacity for 15 calls. In turn, the capacities of crosslinks among LEO satellites is set to be 5 and 10 in the simulations for Figure 4(a) and 4(b), respectively. One can easily observe that the optimal threshold value does depend on the traffic load as well as link capacities. In an operational network, similar figures should be instrumental for tuning the alternate routing algorithm.

Next we investigate the network performance under single or multiple LEO satellite failures. Depicted in Figure 5 are the call blocking probabilities versus traffic loads, with the operating conditions ranging from normal to 3 LEO satellite failures. When multiple LEO satellite failures occur, we assume these satellites to be remotely located. The following system parameters are also assumed: $L E O \_C A P=5, G E O \_C A P=100, L G \_C A P=20$, and threshold $=4$. The simulation results shown in Figure 5 indicate that blocking probabilities in- 
crease only slightly when a small number of LEO satellite failures occur. We note that such increases are mainly due to the destination blocking, as it should be under the assumption of uniform destination distribution. In other words, we have verified the conjecture that high network throughput can be maintained by the alternate routing policy, even under a small-scale failure condition.

\section{CONCLUSIONS}

In this paper, we have proposed a multiple satellite architecture consisting of LEO and GEO satellites as well as an alternate routing policy for circuitswitched communications. We have used simulations to illustrate the network performance in terms of call blocking probability, and have found the advantage of this LEO-GEO network architecture over the multiple LEO satellite network. It is found that the hop count threshold used in the alternate routing is essential to the network performance and its optimal value is traffic load dependent. Finally, simulation results have indicated that a small number of LEO satellite failures could only slightly affect the network performance.

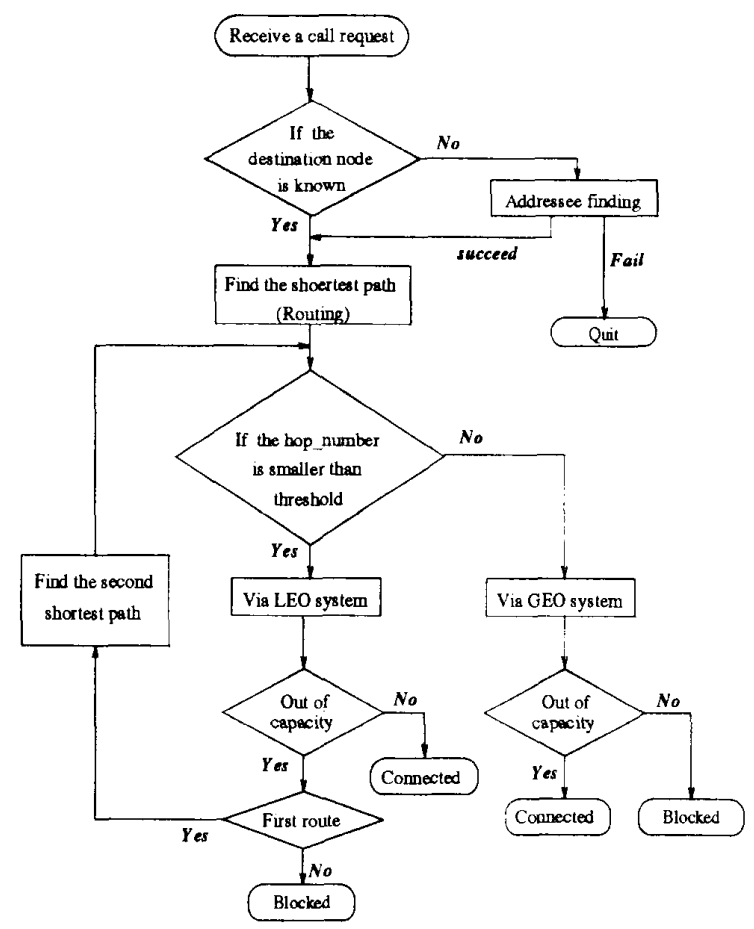

Fig. 1 Flow diagram for the alternate routing algorithm with thresholding

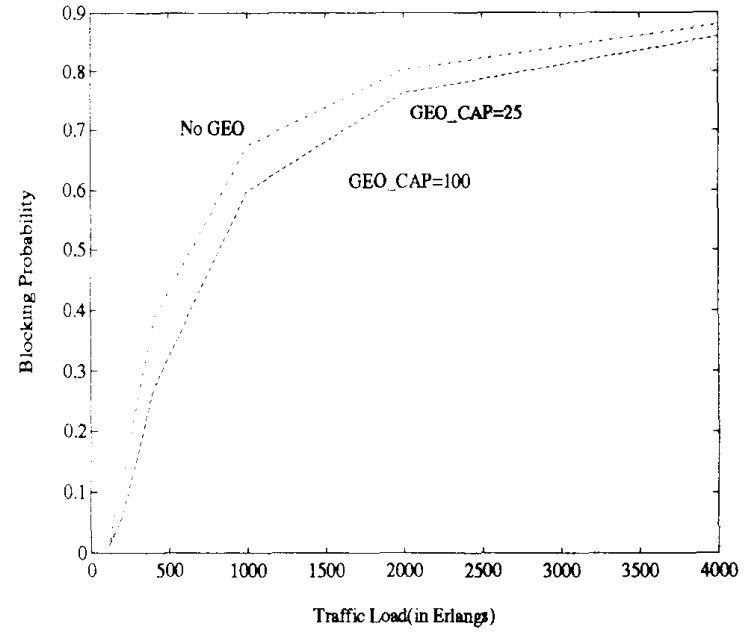

Fig. 2 Call blocking probability versus total network traffic load, with or without GEO satellites, with GEO_CAP $=25$, LEO_CAP $=5$, LG_CAP $=5$, threshold $=4$.

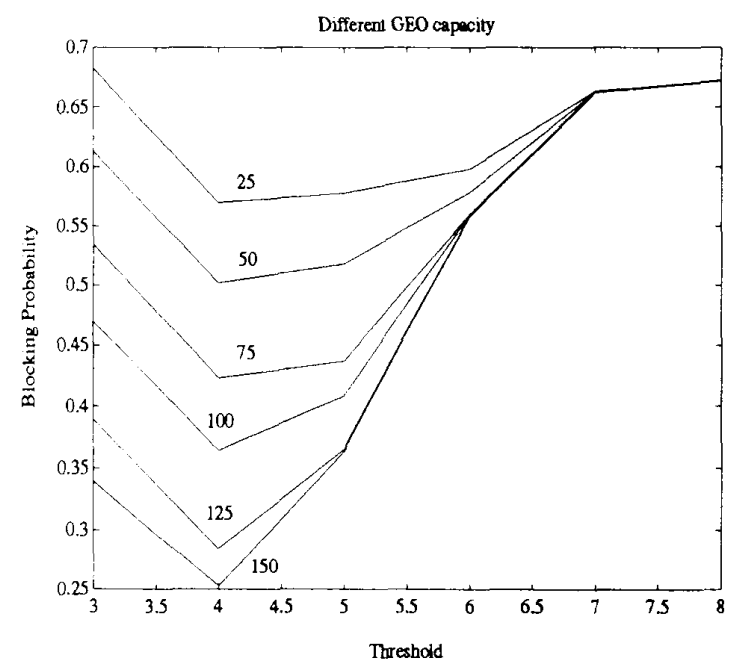

Fig. 3 Call blocking probability versus hop count threshold, with LEO_CAP $=5$, total network traffic load $=1000 \mathrm{Er}$ langs. 


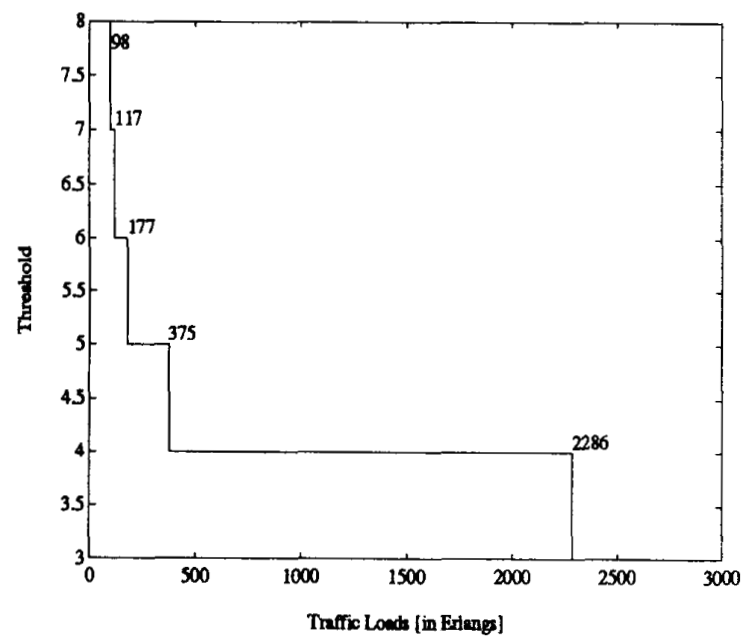

Fig. 4(a) Optimal threshold versus network traffic loads, with LEO_CAP $=5$, GEO_CAP $=75$, and LG_CAP $=15$.

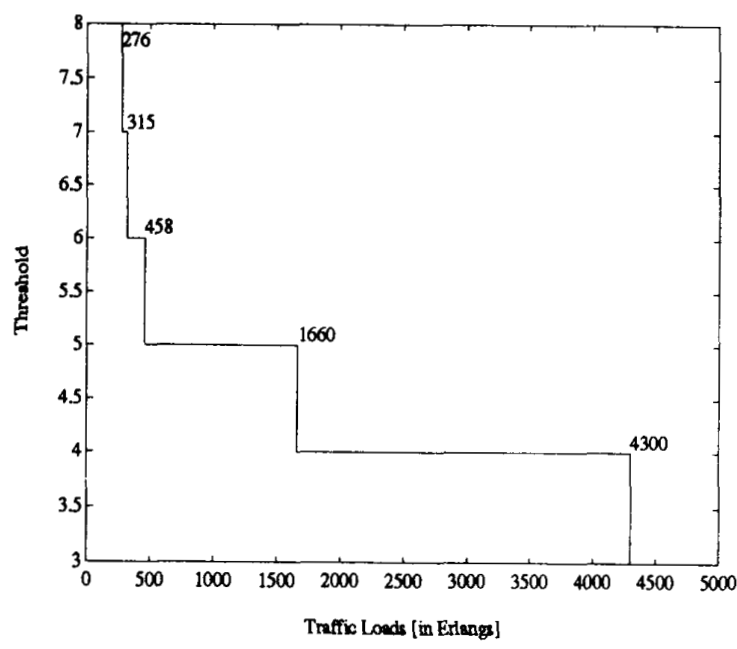

Fig. 4(b) Optimal threshold versus network traffic loads, with LEO_CAP $=10$, GEO_CAP $=75$, and LG_CAP $=15$.

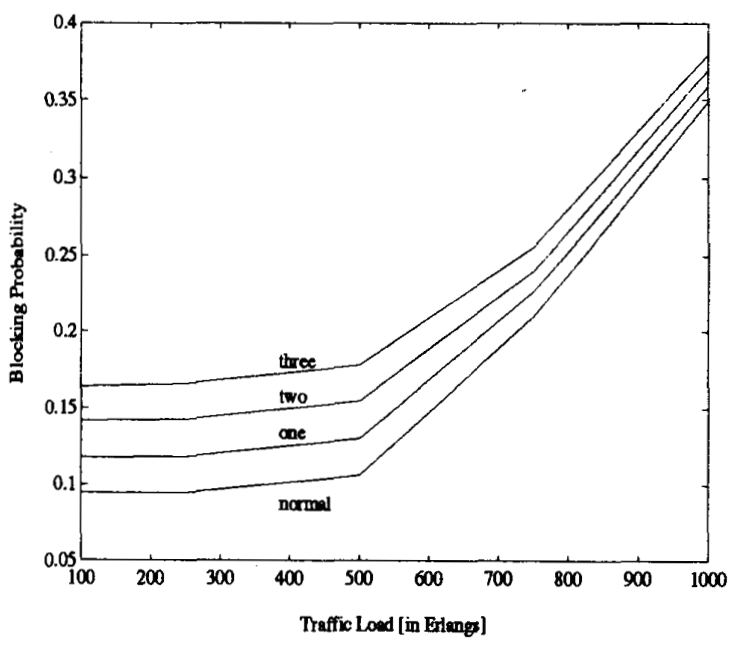

Fig. 5 Call blocking probability versus network traffic load under various LEO satellite failure conditions.

\section{REFERENCES}

[1] K. Brayer, "Packet Switching for Mobile Earth Stations Via Low-orbit Satellite Networks," Proc. IEEE, PROC-72, 1627-1636 (1984).

[2] R.I. Leopold, "Low-Earth Orbit Global Cellular Communications Network," Proc. ICC, 35A.2.1-35A.2.4 (1991).

[3] R. Binder, S.S. Huffman, I. Gurantz, and P.A. Vena, "Crosslink Architecturefor a Multiple Satellite System," Proc. IEEE, PROC-75, (1987).

[4] J. Kaniyil, J. Takei, S. Shimamoto, Y. Onozato, T. Usui, I. Oka and T. Kawabata, "A Global Message Network Employing Low EarthOrbiting Satellites," IEEE J. Selected Areas Communi., SAC-10, 418-427 (1992).

[5] R.S. Krupp, "Stabilization of Alternate Routing Networks," Proc. ICC, 31.2.1-31.2.5 (1982).

[6] A. Girard, "Routing and Dimensioning in Circuit-Switched Networks," Addison Wesley, 1990. 